\title{
Atomic Force Microscopy: Methods and Applications
}

MZ Baykara, Bilkent University, Ankara, Turkey

UD Schwarz, Yale University, New Haven, CT, United States

(c) 2016 Elsevier Inc. All rights reserved.

\begin{tabular}{ll}
\hline Introduction & 1 \\
Methods and Instrumentation & 1 \\
Contact Mode & 2 \\
Tapping Mode & 3 \\
Noncontact Mode & 3 \\
Force Spectroscopy & 3 \\
Applications of Atomic Force Microscopy & 4 \\
Atomic-Resolution Force Spectroscopy in Three Dimensions & 4 \\
Biological Research With the AFM & 4 \\
Nanotribology Studies With the AFM & 5 \\
AFM for Catalysis Research & 5 \\
Outlook on Emerging Aspects and Future Prospects of Atomic Force Microscopy & 6 \\
\hline
\end{tabular}

\begin{tabular}{|c|c|c|}
\hline Symbols & $F$ & tip-sample interaction force \\
\hline oscillation amplitude & $f_{0}$ & resonance frequency \\
\hline$A_{\text {exc }}$ excitation amplitude & $k$ & spring constant \\
\hline$d$ tip-sample distance & $\varphi$ & phase \\
\hline$\Delta A$ amplitude shift & $V$ & bias voltage \\
\hline
\end{tabular}

\section{Introduction}

The invention of scanning probe microscopes (SPMs) in the 1980s and their rapid expansion to academic and industrial laboratories is a key contributor enabling the recent rise of nanoscience and nanotechnology. Unlike averaging spectroscopic techniques such as X-ray photoelectron spectroscopy, SPMs allow the local characterization of material surfaces in real space. In particular, it has become possible to obtain atomic-resolution structural maps of surfaces and measure associated physical and chemical properties with site-specific precision using the so-called spectroscopy modes, leading to the recording of crucial fundamental information relevant for a large variety of scientific fields including, but not limited to, device research, thin film growth, heterogeneous catalysis, friction, and biology.

All SPMs are operated via the utilization of a very sharp (under ideal cases, atomically sharp) probe tip that either (i) forms a slight contact with, or (ii) is brought into very close proximity (on the order of several nanometers or below) of the surface to be investigated. The probe tip is raster-scanned over the surface, and the interaction of the tip with the sample in the form of, for example, tunneling currents or interaction forces is recorded. Employing feedback loops to keep relevant physical quantities constant during scanning leads to the acquisition of high-resolution topographic maps of surfaces, providing real-space information on structure including defects such as step edges, domain boundaries, vacancies, and adsorbed species. Moreover, spectroscopy experiments where tip-sample interactions are recorded as a function of tip-sample distance and other experimental parameters such as sample bias are conducted to elucidate local chemical, electronic, and mechanical properties.

Out of the various types of SPMs available today, the atomic force microscope (AFM) is by far the most widely employed, mainly due to its versatility in terms of materials to which it may be applied regardless of electrical conductivity (metals, metal oxides, semiconductors, etc.) as well as the variability in operating environments, ranging from ambient and liquids to ultrahigh vacuum (UHV). In this chapter, the basic instrumentation and most common operational modes of AFM will be reviewed. Additionally, various applications of the method will be exemplified via the reporting of illustrative results from the literature. The chapter will conclude with an outlook on emerging aspects and future prospects of AFM research. 


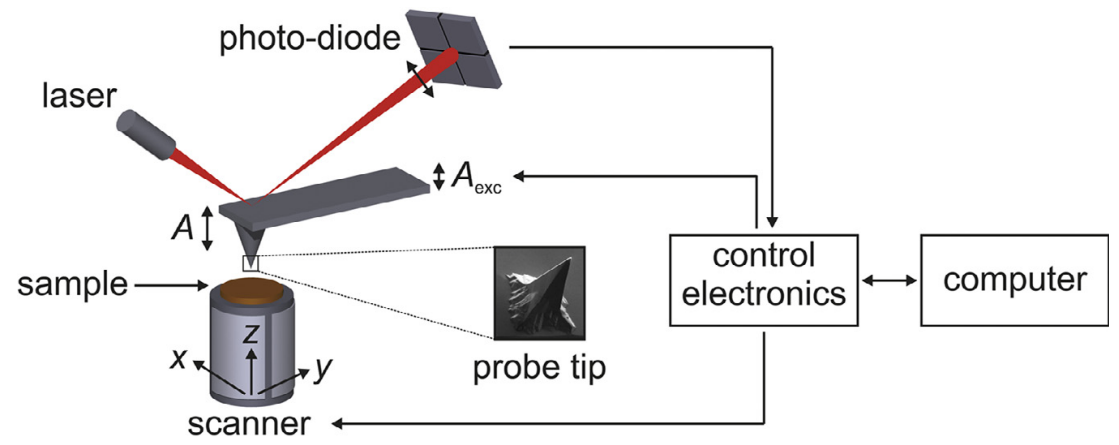

Fig. 1 Schematic drawing of a typical AFM setup. A piezoelectric scanner is utilized to control the relative tip-sample position with picometer precision in three dimensions, while the deflections of a micro-fabricated cantilever with integrated probe tip are detected via laser beam deflection. A computer is used to drive control electronics responsible for tip-sample positioning, data acquisition, and the operation of feedback loops. For dynamic AFM operation, the cantilever base is excited with an amplitude $A_{\text {exc }}$, resulting in an oscillation of the probe tip with amplitude $A$. Adapted from Baykara, M. Z., et al. (2010) Adv. Mater. 22, 2838-2853.

\section{Methods and Instrumentation}

The basic operational principle of the AFM relies on the precise detection of interaction forces acting between a sharp probe tip and a sample surface at close separations. To perform imaging, the probe tip is raster-scanned over the sample surface while the interaction force $F$ or a related physical quantity is held constant via feedback loops. To perform spectroscopy, the interaction force $F$ or the related physical quantity can be measured as a function of various experimental parameters, such as tip-sample distance $d$ and bias voltage $V$.

The key components that constitute an AFM (Fig. 1) can be identified as:

(i) A scanner to position the sample with respect to the probe tip with picometer-scale precision in three dimensions $(x, y, z)$;

(ii) A force sensing element and the associated detection scheme;

(iii) Control electronics and a computer to (a) control scanners and other components; (b) record, display, and save data; and (c) operate feedback loops.

Scanners that are widely utilized in AFM setups are made of piezoelectric materials in various geometries that elongate/contract with applied voltage. While using flexure stages employing piezoelectric elements typically allows positioning in the lateral $x, y$ directions a single scanner in the shape of a piezoelectric tube with five isolated electrodes allows tip-sample positioning in the lateral $x, y$ as well as vertical $z$ directions. All scanners made of piezoelectric elements are subject to nonlinearities including hysteresis and creep, which are alleviated to a certain extent in modern AFMs using software- and/or hardware-based correction schemes.

The force-sensing element in a modern AFM typically consists of a micro-fabricated cantilever $\left(\mathrm{Si}, \mathrm{SiO}_{2}\right.$, or $\mathrm{Si}_{3} \mathrm{~N}_{4}$, with a length on the order of a few hundreds of micrometers) with an integrated, sharp tip. The cantilever acts as a soft spring (spring constants $k$ ranging from $\sim 0.01$ to $\sim 100 \mathrm{Nm}^{-1}$ ), deflecting in response to the force interaction $F$ between the tip and the sample. In recent years, the use of piezoelectric quartz tuning forks instead of micro-machined cantilevers (with $k$ values on the order of $\sim 2000 \mathrm{Nm}^{-1}$ ) has been rapidly increasing, specifically in microscope setups geared toward atomic-resolution imaging.

Deflections of the force sensor are detected via three main approaches:

(i) using a laser beam deflected off the force sensor and collected on a photo-diode;

(ii) using an interferometer brought into close proximity of the force sensor;

(iii) electrically via the utilization of piezoresistive cantilevers or piezoelectric tuning forks.

Modern AFMs are commonly operated in three distinct modes, details of which are discussed in the following sections: the "static" mode (frequently referred to as contact mode), and two "dynamic" modes, where the force sensor is oscillated at or near its resonance frequency using a piezoelectric element with excitation amplitude $A_{\text {exc }}$ (tapping mode and noncontact mode).

\section{Contact Mode}

Contact mode imaging is the simplest and the most straightforward way of operating an AFM. The probe tip, which is located at the end of a very soft cantilever ( $<1 \mathrm{Nm}^{-1}$ typically), is first brought into close proximity of the sample surface, with the tip eventually "snapping" into contact with the surface at the moment the gradient of the tip-sample interaction overcomes the low spring constant of the force sensor. The repulsive interaction force that acts between the tip and the sample is then monitored via detecting the associated deflection of the force sensor and is kept constant at a fixed value (typically on the order of a few nanonewton) via a feedback loop as the tip is raster-scanned over the surface. Tracking and recording the vertical position of the force sensor base with respect to the surface leads to the acquisition of topographical maps with nanometer-scale resolution. Moreover, recording the twisting of the cantilever during scanning due to the torque caused by the lateral forces acting on the tip apex allows quantitative 
measurements of friction, with the possibility of studying its dependence on various physical parameters for the nanometer-scale contact geometry realized by the tip apex and the sample surface.

Despite their operational simplicity, contact mode AFM experiments have certain disadvantages, most notably an intrinsically low signal-to-noise ratio compared to "dynamic" modes tracking modulated signals, and the fact that lateral forces and repulsive interactions may damage the tip apex and the sample surface during scanning.

\section{Tapping Mode}

Shortly after the invention of the AFM, efforts were directed at overcoming the drawbacks associated with contact mode operation and increasing the amount of physical information that can be extracted from experiments. This led to the introduction of "dynamic" operation modes, which involve the deliberate excitation of the force sensor at or near its resonance frequency $f_{0}$ with excitation amplitude $A_{\text {exc }}$. When the tip is brought close to the sample surface, oscillation characteristics (frequency, amplitude, phase) change in response to tip-sample interaction forces. In the so-called tapping mode, the force sensor is driven with a fixed frequency and changes in its amplitude A (commonly on the order of several up to tens of $\mathrm{nm}$ ) and phase $\varphi$ are tracked as a function of tip-sample position. Keeping the change in amplitude $\Delta A$ (and thus the tip-sample force interaction) constant during raster-scanning leads to the acquisition of topographical maps with nanometer-scale resolution without adverse effects associated with permanent repulsive contact and lateral forces causing tip and sample damage. As such, tapping mode imaging is frequently employed to characterize soft biological materials, as well as other samples, mostly in air and liquids. It is important to note that the relatively large amplitudes used during tapping mode cause the tip to cover both attractive and repulsive parts of the tip-sample interaction, thus resulting in the apex periodically "tapping" the surface during operation, or in other words, the formation of intermittent tip-sample contact. Consequently, the $\varphi$ data that are acquired during tapping mode imaging include information about the nanomechanical properties of the sample surface. Due to its focus on amplitude changes as a feedback parameter, tapping mode AFM is alternatively referred to as "amplitude modulation" AFM (AM-AFM).

\section{Noncontact Mode}

A fundamental shortcoming of both contact and tapping mode AFM involves the formation of a finite contact area between the tip apex and the sample surface during operation. Thus, a tip that is atomically sharp at the onset of the experiment becomes blunt during operation, resulting in the inability to achieve atomic resolution via contact and tapping mode AFM in most cases. To achieve atomic-resolution imaging, the dynamic operation mode of "noncontact" AFM (NC-AFM) has been introduced. In NC-AFM, the force sensor is initially oscillated at its resonance frequency $f_{0}$ and brought into close proximity of the sample surface. In contrast to tapping mode, the oscillation amplitude is kept constant at small values (ranging from $\approx 1 \AA$ to a few nanometers) such that the interaction can be restricted to the attractive regime. Thus, "contact" between tip and sample (as defined by the occurrence of repulsive interactions) is avoided, and tips that are atomically sharp remain so during the experiment. Rasterscanning with a constant change in resonance frequency $(\Delta f)$ may, under optimum conditions, then lead to the acquisition of topography maps with atomic resolution; due to the use of $\Delta f$ for distance regulation, this operational mode is also denoted as "frequency modulation" AFM (FM-AFM). Despite the fact that the proper utilization of the NC-AFM method is not as straightforward as contact and tapping mode (mainly due to the multiple feedback loops that the technique necessitates), impressive results have been demonstrated in recent years, including but not limited to the atomic-scale characterization of insulating metal oxide surfaces, the visualization of the intramolecular structure of adsorbates, and the discrimination of chemical bond order.

\section{Force Spectroscopy}

In addition to above-mentioned imaging modes, AFM is capable of performing force spectroscopy, involving the experimental measurement of the interaction force $F$ or a related physical quantity (such as $\Delta A$ in tapping mode or $\Delta f$ in noncontact mode) at well-defined positions on a sample surface as a function of various physical parameters such as tip-sample distance $d$ or bias voltage $V$.

In particular, $F$ vs. $d$ curves acquired with a static cantilever allow the investigation of local sample stiffness (via the determination of the slope of the $F$ vs. $d$ curve in the repulsive regime) and the quantification of tip-sample adhesion (via the determination of minima observed in $F$ vs. $d$ curves as the tip is first brought into contact and then retracted from the surface). Note that due to the "snapping into contact" of the cantilever upon approach, static force spectroscopy measurements will not yield information on attractive interactions at very close tip-sample distances.

The latter drawback is overcome using dynamic AFM modes. Even though they track $\Delta f$ (for NC-AFM) or $\Delta A$ and $\varphi$ (for tappingmode AFM) rather than directly $F$, such data can be converted to $F$ post-data-acquisition via analytical methods if complete data sets for $\Delta f(z)$ or $A(z)$ and $\varphi(z)$, respectively, are recorded up to tip-sample distances where the interaction exerted by the surface onto the tip has dropped below the detection limit. 


\section{Applications of Atomic Force Microscopy}

\section{Atomic-Resolution Force Spectroscopy in Three Dimensions}

Thanks to the capability of the NC-AFM method to deliver atomic-resolution maps, $F(\mathrm{z})$ curves can be acquired in this mode with atomic precision using the force spectroscopy approach introduced above. Combining thousands or even tens of thousands of these curves into a single data array has recently led to the development of "three-dimensional" AFM (3D-AFM), which has resulted in the acquisition of three-dimensional force spectroscopy maps with pm- and pN-resolution on surfaces including graphite and metal oxides as well as adsorbed molecules such as pentacene and $\mathrm{C}_{60}$. Additional data channels such as tunneling current and dissipation (as calculated by the increase in $A_{\text {exc }}$ required to keep $A$ constant) may be simultaneously recorded, providing complementary information about atomic-scale surface properties.

A particularly illustrative example of atomic-resolution force spectroscopy has been demonstrated on a surface-oxidized $\mathrm{Cu}(100)$ sample where site-specific tip-sample interaction forces and tunneling currents have been recorded (Fig. 2). Specifically, it has been established that simultaneous imaging of multiple atomic species (oxygen atoms in the force channel, copper atoms in the tunneling current channel) can be accomplished via such an approach. Significant variations in interaction forces have been detected on individual oxygen atoms which has been attributed to the variability in the electronic and chemical environment caused by the existence of linear surface defects observed in the tunneling current channel. Lastly, comparison of experimental data with ab initio simulations resulted in the chemical and structural identification of the tip apex and the surface defects.

Further accomplishments of atomic-resolution force spectroscopy include mapping of the force field associated with adsorbed molecules on surfaces, investigating the angular symmetry and order of chemical bonds, three-dimensional mapping of molecular order at solid-liquid interfaces and obtaining pm- and pN-resolution maps of lateral interaction forces. Finally, the charge state of defects on single crystal surfaces as well as the intramolecular distribution of charge inside individual adsorbates can be probed via the method of Kelvin probe force microscopy (KPFM), based on spectroscopy data involving the dependence of $\Delta f$ on $V$.

\section{Biological Research With the AFM}

One of the major advantages associated with the AFM is the fact that it can be performed under operating conditions ranging from UHV to ambient and liquids. The ability of the AFM to deliver high-resolution topographical maps of material surfaces outside of vacuum is of great importance for biology, as it presents the opportunity to study biological materials in "close-to-natural" conditions without loss of structure and function that is unavoidable when they are investigated under vacuum via electron microscopy. Consequently, a large number of biological materials, including cells, DNA, lipid bilayers, as well as fibrous and globular proteins, have been investigated using a variety of operating modes under diverse environmental conditions since the method was first introduced.

It is important to realize that not only imaging but also force spectroscopy experiments can be performed on biological materials via AFM to study associated nanomechanical properties. For example, $F$ vs. $d$ spectroscopy experiments performed on human breast biopsies have recently helped establish that the stiffness characteristics associated with healthy, benign, and malignant tissues vary significantly within the context of breast cancer, providing a nanomechanical signature with potential diagnostic implications.

While the distance dependence of the tip-sample interaction force can be studied to draw conclusions about nanomechanical properties of biological materials, it has been shown that the temporal characteristics of the tip-sample interaction during tapping mode operation can be exploited to discriminate DNA sequences. In fact, when single-stranded DNAs are used as markers for binding sites within an individual protein that is probed with an AFM tip functionalized with complementary DNAs in a liquid environment, time-dependent force spectroscopy allows locating individual binding sites within the protein (Fig. 3).
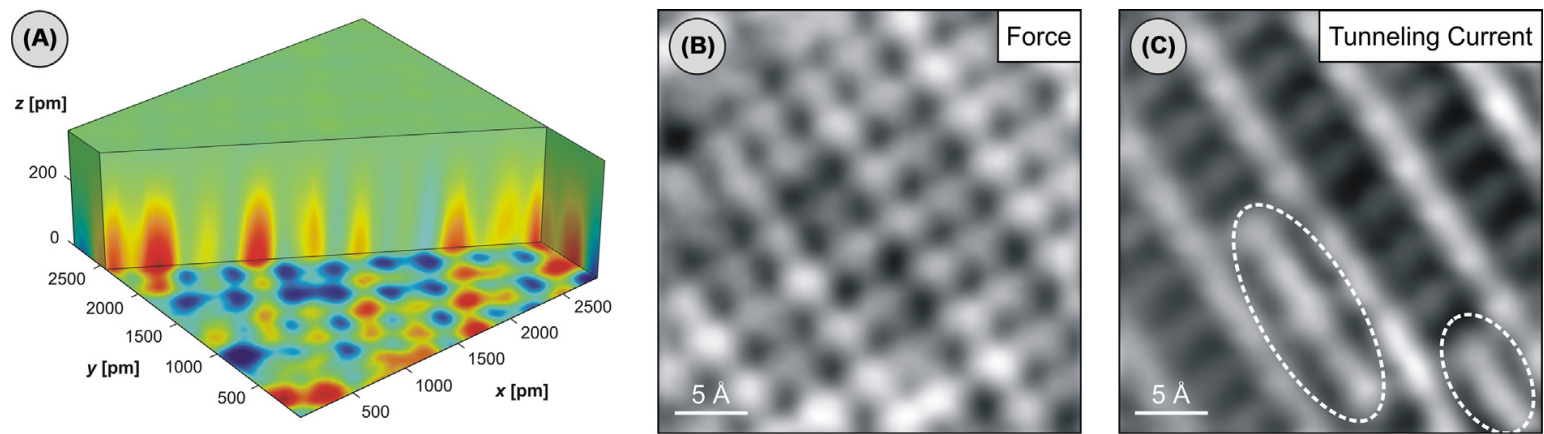

Fig. 2 (A) Three-dimensional, atomic-resolution force array obtained on surface-oxidized copper via 3D-AFM. Total force corrugation (most attractive/ dark red to least attractive/dark blue) is $\approx 23 \mathrm{pN}$. (B) Horizontal force map extracted from (A) at the closest tip-sample distance. Variation of interaction force on individual 0 atoms is observable. (C) Simultaneously recorded tunneling current map. The contrast is dominated by Cu atoms. Defects are highlighted. Adapted from Baykara, M. Z., et al. (2013) Phys. Rev. B 87, 155414. 
(A)

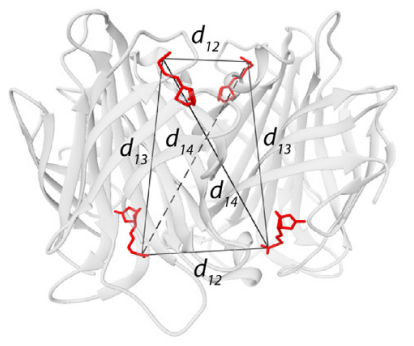

(B)

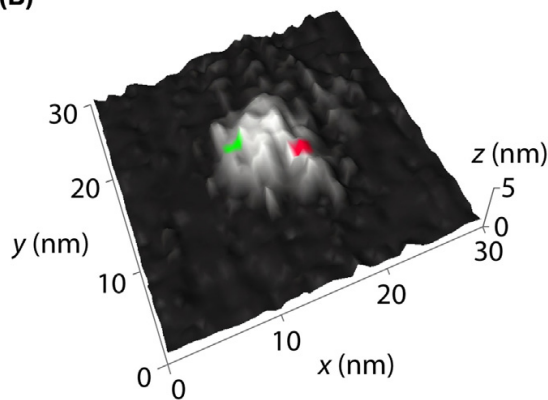

Fig. 3 (A) Model of the crystal structure of the protein streptavidin, with four biotins (shown in red) attached to binding sites as anchors for DNA markers. Three distinct distances between biotin pairs are shown with $d_{12}, d_{13}$, and $d_{14}$. (B) Three-dimensional representation of an individual streptavidin-biotin complex, obtained in liquid via tapping mode AFM with a DNA-functionalized probe tip. The colored dots mark locations of "rupture" events as detected by temporal force spectroscopy, corresponding to the locations of DNA-marked binding sites inside the protein. Reprinted by permission from Macmillan Publishers Ltd: Kim, D. and Sahin, 0. (2015) Nat. Nanotechnol. 10, 264-269, copyright (2015).

\section{Nanotribology Studies With the AFM}

As discussed in the section "Methods and Instrumentation", tracking the twisting of the AFM cantilever due to lateral forces during contact mode operation allows the study of friction on the nanometer scale, thus opening the way to examine its dependence on various physical parameters such as normal force, sliding velocity, contact area, and temperature for the well-defined contact geometry realized by the AFM tip apex and the sample surface. Such experiments are often referred to as "friction force spectroscopy." Considering that the fundamental physical principles governing friction are still not fully understood and that macroscopic contacts are very difficult to experimentally analyse due to the fact that they consist of multiple asperities, fundamental studies of friction performed via AFM have the potential to contribute significantly to related efforts.

Motivated by the discussion above, numerous studies of friction have been performed via AFM since its invention as part of a research field referred to as "nanotribology." In particular, it has been determined that friction on the nanometer scale often features a stick-slip character, where the motion of the AFM tip above the sample surface is not continuous but characterized by the tip apex periodically getting trapped in and jumping between the minima of the tip-sample interaction potential (Fig. 4A-C). Furthermore, phenomena such as superlubric sliding (i.e., sliding with a frictional resistance that may be orders of magnitude lower than conventionally expected) have been observed, for instance, between individual graphite flakes. Frictional properties of two-dimensional materials such as graphene have also been studied via AFM, where it has been demonstrated that the friction exhibited by mechanically exfoliated samples of such materials monotonously decreases with increasing layer number, with implications for solid lubrication of nanomechanical systems. Recently, AFM has also been used to laterally manipulate individual nanoparticles (Fig. 4D) on substrates such as graphite to study interfacial friction as a function of particle size, where a linear dependence of friction on contact area has been demonstrated as well as the occurrence of nonlinear superlubric sliding under UHV conditions (Fig. 4E).

\section{AFM for Catalysis Research}

A full understanding of heterogeneous catalysis requires experimental access to the atomic-scale properties of catalytic surfaces in real space. For instance, catalytic processes on metal oxide surfaces are mainly governed by the electronic and structural properties of surface defects including vacancies and step edges. Limited electrical conductivities of most metal oxide surfaces relevant for catalysis prevent the utilization of experimental tools such as the scanning tunneling microscope. As such, AFM arises as the ideal method to perform related investigations with its capability of site-specific spectroscopy of tip-sample interactions with atomic resolution as a function of tip-sample position and bias voltage. 
(A)

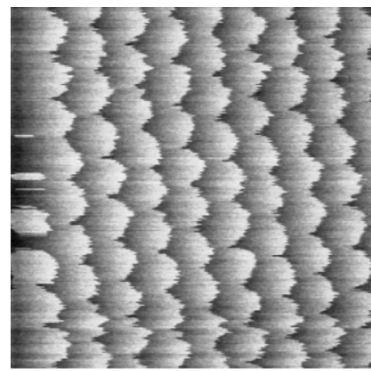

(D)
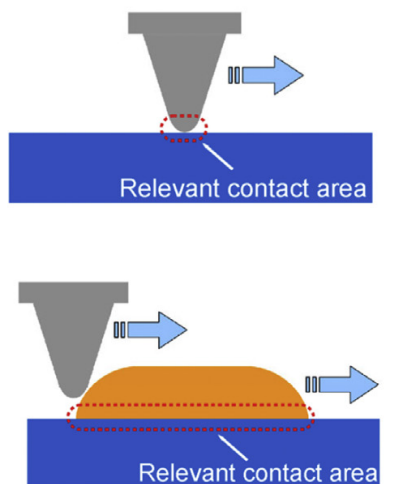

(B)

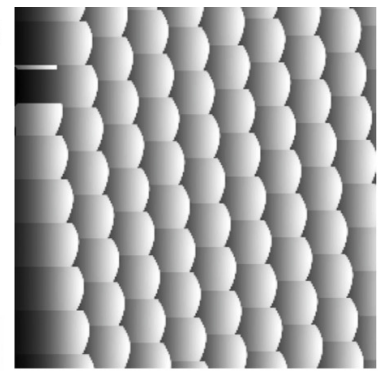

(C)

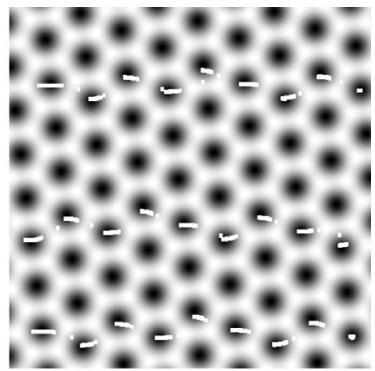

(E)

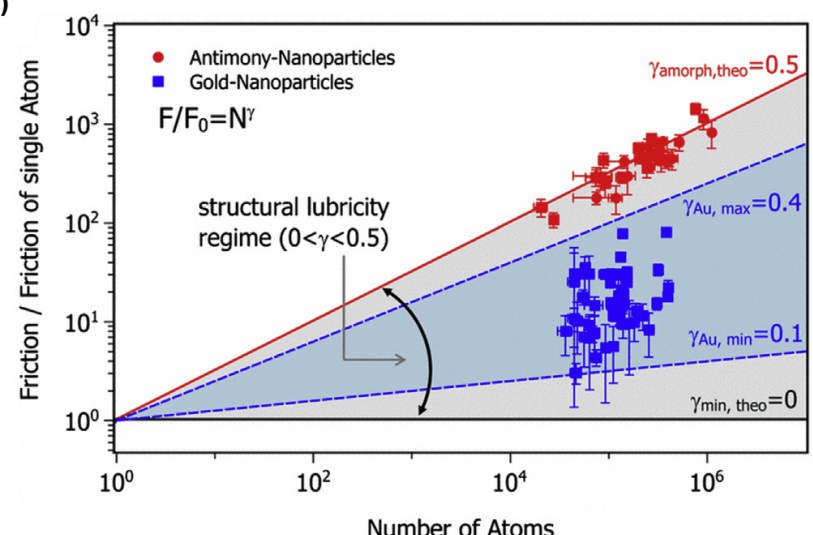

Fig. 4 Experimental (A) and simulated (B) friction force maps obtained on graphite via AFM, highlighting stick-slip behavior. (C) Map of the interaction potential used for the simulations where three tip paths reconstructed from data in $(A)$ are indicated by white dots; each dot represents the position of the tip at specific times separated by equal intervals of $0.15 \mathrm{~ms}$. (D) Schematic drawings describing the relevant contact areas for conventional nanotribology experiments (top) and those that involve lateral manipulation of nanoparticles (bottom). (E) Results of manipulation experiments conducted with Au and Sb nanoparticles sliding on atomically flat, clean graphite terraces under UHV conditions, confirming superlubric sliding with sub-linear dependence of friction on contact area. Reproduced from the following references: Hölscher, H., et al. (1998) Physical Review $B$ 57, 2477-2481 (A-C); Dietzel, D., et al. (2014) Friction 2, 114-139 (D); and Dietzel, D., et al. (2013) Phys. Rev. Lett. 111, 235502 (E).

Multiple experiments have been reported in the literature where AFM has been used to investigate the atomic-scale structure and reactivity of bare catalytic surfaces (including $\mathrm{Al}_{2} \mathrm{O}_{3}, \mathrm{CeO}_{2}$, and $\mathrm{TiO}_{2}$ ) as well as adsorbates such as organic molecules and metal atoms. In particular, NC-AFM has been utilized to perform spectroscopy experiments involving the measurement of the frequency shift (directly related to the tip-sample interaction force) as a function of both position and bias voltage on the model $\mathrm{MgO} / \mathrm{Ag}(001)$ system, allowing one to distinguish point defects with different charge states and also to determine the strong attractive character of their chemical interaction with the metallic tip apex (Fig. 5).

\section{Outlook on Emerging Aspects and Future Prospects of Atomic Force Microscopy}

While the representative examples of AFM applications presented in this chapter constitute an overview of the state-of-the-art for this exciting method, the number of material systems and scientific issues where AFM can provide crucial results is rapidly expanding.

Examples of areas of emerging importance include:

(i) Standardization of the chemistry and structure of probe tips via functionalization using molecules such as $\mathrm{CO}$;

(ii) High-resolution measurements of structure and chemistry associated with various materials in liquids;

(iii) High-speed measurements of dynamic processes, in particular those associated with biomolecules;

(iv) Atom-by-atom assembly of nano-scale functional structures on surfaces;

(v) Submolecular-resolution, three-dimensional imaging of adsorbates such as $\mathrm{C}_{60}$.

Considering the wide range of scientific and technological problems to which it can be applied and its flexibility in terms of operating conditions and material requirements, it is projected that AFM in its various modes of operation will continue to be the most extensively used scanning probe method for the foreseeable future. With increasing standardization in terms of the physical characteristics of the probe tip and further simplification of operation associated with specialized modes such as NC-AFM, the industrial use of the method is also expected to escalate. 

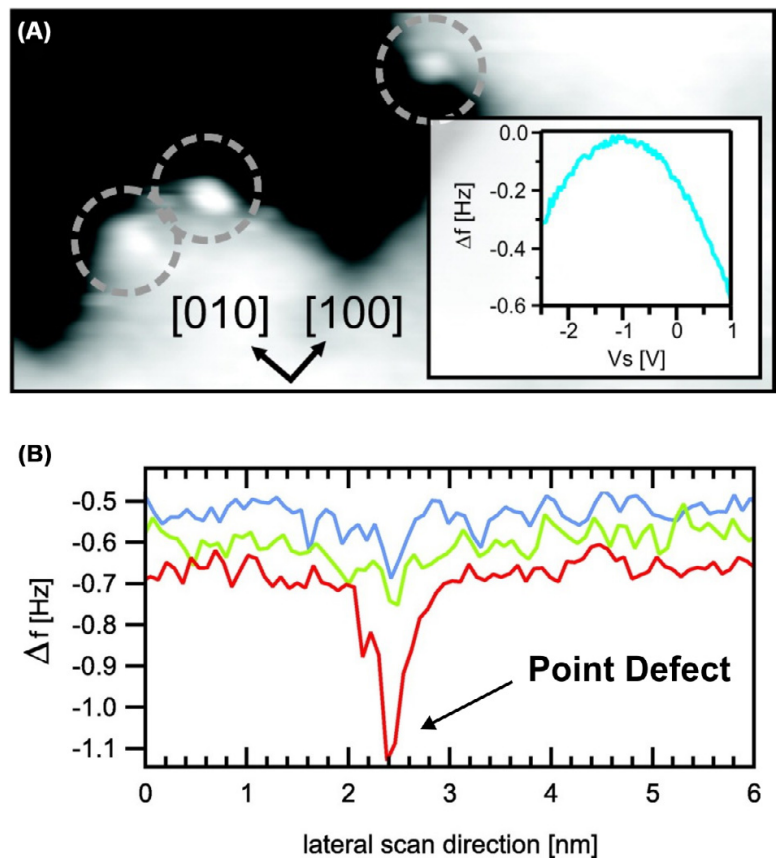

Fig. 5 (A) NC-AFM image of an $\mathrm{MgO}$ step edge on $\mathrm{Ag}(001)$; point defects are highlighted. A frequency shift vs. bias voltage spectroscopy curve acquired on a defect is presented in the inset, facilitating the determination of the associated charge state. (B) Frequency shift data recorded along a horizontal line passing over a point defect at three different tip-sample distances separated by $0.5 \AA$, with the red curve corresponding to the smallest separation. Negative frequency shift values are detected at the defect site, indicative of attractive tip-sample interactions. Reprinted with permission from König, T., et al. (2009) J. Am. Chem. Soc. 131, 17544-17545 (A) and König, T., et al. (2010) ACS Nano 4, $2510-2514$ (B). Copyright (2009) and (2010) American Chemical Society.

\section{Further Reading}

1. Baykara, M. Z. In Surface Science Tools for Nanomaterials Characterization; Kumar, C. S. S. R., Ed.; Springer: Berlin, 2015; pp 273-316.

2. Baykara, M. Z.; Schwendemann, T. C.; Altman, E. I.; Schwarz, U. D. Adv. Mater. 2010, 22, 2838-2853.

3. Dietzel, D.; Schwarz, U. D.; Schirmeisen, A. Friction 2014, 2, 114-139.

4. Eaton, P. J.; West, P. Atomic Force Microscopy; Oxford University Press: Oxford, 2010.

5. Garcia, R. Amplitude Modulation Atomic Force Microscopy; Wiley-VCH: Weinheim, 2010

6. Giessibl, F. J. Rev. Mod. Phys. 2003, 75, 949-983.

7. Gross, L.; Mohn, F.; Moll, N.; Liljeroth, P.; Meyer, G. Science 2009, 325, 1110-1114.

8. Hölscher, H.: Schirmeisen, A.: Schwarz, U. D. Phil. Trans. R. Soc. A 2008, 366, 1383-1404.

9. Kodera, N.; Yamamoto, D.; Ishikawa, R.; Ando, T. Nature 2010, 468, 72-76.

10. Lauritsen, J. V.; Reichling, M. J. Phys. Condens. Matter 2010, 22, 263001

11. Morita, S.; Giessibl, F. J.; Meyer, E.: Wiesendanger, R., Eds.; Noncontact Atomic Force Microscopy; vol. 1-3: Springer: Berlin, 2002-2015.

12. Morris, V. J.; Kirby, A. R.; Gunning, A. P. Atomic Force Microscopy for Biologists; Imperial College Press: London, 2009.

13. Plodinec, M.; et al. Nat. Nanotechnol. 2012, 7, 757-765.

14. Seo, Y.: Jhe, W. Rep. Prog. Phys. 2008, 71, 016101

15. Urbakh, M.; Meyer, E. Nat. Mater. 2010, 9, 8-10. 